\title{
Exact Harmonic Metric for a Moving Reissner-Nordström Black Hole
}

\author{
G. He and W. Lin* \\ School of Physical Science and Technology, \\ Southwest Jiaotong University, Chengdu 610031, China
}

\begin{abstract}
The exact harmonic metric for a moving Reissner-Nordström black hole with an arbitrary constant speed is presented. As an application, the post-Newtonian dynamics of a non-relativistic particle in this field is calculated.
\end{abstract}

PACS numbers: 04.20-q, 04.20.Jb, 04.70.Bw, 95.30.Sf

\footnotetext{
* To whom correspondence should be addressed. Email: wl@ swjtu.edu.cn
} 


\section{INTRODUCTION}

The motion of a gravitational source can affect the dynamics of particle passing by it, and this effect has attracted considerable attention over the last two decades [1-12]. There are several methods to calculate this effect. One is to directly solve Liénard-Wiechert gravitational potential from the field equations, which has been used to study light propagation in the gravitational field of an arbitrarily moving N-body system, as well as that with angular momentum [3, 5]. Another method takes advantage of the general covariance of field equations to obtain the metric of the moving source from the known static source's metric via Lorentz transformation [13]. Recently, this method was employed to derive the time-dependent harmonic metrics of arbitrary-constant moving Schwarzschild and Kerr black holes [14-16].

In this work, we apply a Lorentz transformation to derive the exact harmonic metric for a moving Reissner-Nordström black hole with an arbitrary constant speed. Furthermore, based on the metric, we calculate the dynamics of a photon and a particle in the weak-field limit. In what follows we use geometrized units $(G=c=1)$.

\section{EXACT HARMONIC METRIC FOR AN ARBITRARILY CONSTANTLY MOVING REISSNER- NORDSTRÖM BLACK HOLE}

We start with the harmonic metric of Reissner-Nordström black hole, which can be written as [17]

$$
d s^{2}=-\frac{R^{2}-m^{2}+Q^{2}}{(R+m)^{2}} d X_{0}^{2}+\left(1+\frac{m}{R}\right)^{2}\left[\delta_{i j}+\frac{m^{2}-Q^{2}}{R^{2}-m^{2}+Q^{2}} \frac{X_{i} X_{j}}{R^{2}}\right] d X_{i} d X_{j},
$$

where $m$ and $Q$ are the rest mass and electric charge of the black hole, respectively. $i, j=$ $1,2,3$, and $\delta_{i j}$ denotes Kronecker delta. Notice that here $X_{\mu}$ denotes the contravariant vector $x^{\prime \mu}=\left(t^{\prime}, x^{\prime}, y^{\prime}, z^{\prime}\right)$ for display convenience, and $R^{2}=X_{1}^{2}+X_{2}^{2}+X_{3}^{2}$.

Since Einstein field equations have the property of general covariance, the harmonic metric of a constantly moving R-N black hole can be obtained via applying a Lorentz boost to Eq. (1). We denote the coordinate frame of the background as $(t, x, y, z)$, and assume the velocity of the black hole to be $\boldsymbol{v}=v_{1} \boldsymbol{e}_{1}+v_{2} \boldsymbol{e}_{2}+v_{3} \boldsymbol{e}_{3}$, with $\boldsymbol{e}_{i}(i=1,2,3)$ denoting the unit vector of 3-dimensional Cartesian coordinates. The Lorentz transformation between $(t, x, y, z)$ and the comoving frame $\left(t^{\prime}, x^{\prime}, y^{\prime}, z^{\prime}\right)$ of the moving hole can be written as

$$
x^{\prime \alpha}=\Lambda_{\beta}^{\alpha} x^{\beta},
$$


with

$$
\begin{aligned}
\Lambda_{0}^{0} & =\gamma, \\
\Lambda_{0}^{i} & =\Lambda_{i}^{0}=-v_{i} \gamma, \\
\Lambda_{j}^{i} & =\delta_{i j}+v_{i} v_{j} \frac{\gamma-1}{v^{2}},
\end{aligned}
$$

where $\gamma=\left(1-v^{2}\right)^{-\frac{1}{2}}$ is the Lorentz factor and $v^{2}=v_{1}^{2}+v_{2}^{2}+v_{3}^{2}$. Therefore, the exact harmonic metric of the moving Reissner-Nordström black hole can be obtained as follows

$$
\begin{aligned}
g_{00}= & -\frac{\gamma^{2}\left(R^{2}-m^{2}+Q^{2}\right)}{(R+m)^{2}}+\gamma^{2}\left(1+\frac{m}{R}\right)^{2}\left[\boldsymbol{v}^{2}+\frac{(\boldsymbol{v} \cdot \boldsymbol{X})^{2}\left(m^{2}-Q^{2}\right)}{R^{2}\left(R^{2}-m^{2}+Q^{2}\right)}\right], \\
g_{0 i}= & v_{i} \gamma^{2}\left[\frac{R^{2}-m^{2}+Q^{2}}{(R+m)^{2}}-\left(1+\frac{m}{R}\right)^{2}\right]-\gamma\left(1+\frac{m}{R}\right)^{2} \frac{m^{2}-Q^{2}}{R^{2}\left(R^{2}-m^{2}+Q^{2}\right)} \times \\
& {\left[X_{i}(\boldsymbol{v} \cdot \boldsymbol{X})+\frac{v_{i}(\gamma-1)(\boldsymbol{v} \cdot \boldsymbol{X})^{2}}{\boldsymbol{v}^{2}}\right], } \\
g_{i j}= & \left(1+\frac{m}{R}\right)^{2}\left\{\delta_{i j}+\frac{m^{2}-Q^{2}}{R^{2}\left(R^{2}-m^{2}+Q^{2}\right)}\left[X_{i}+\frac{v_{i}(\gamma-1)(\boldsymbol{v} \cdot \boldsymbol{X})}{\boldsymbol{v}^{2}}\right] \times\right. \\
& {\left.\left[X_{j}+\frac{v_{j}(\gamma-1)(\boldsymbol{v} \cdot \boldsymbol{X})}{\boldsymbol{v}^{2}}\right]\right\}+v_{i} v_{j} \gamma^{2}\left[\left(1+\frac{m}{R}\right)^{2}-\frac{R^{2}-m^{2}+Q^{2}}{(R+m)^{2}}\right] . }
\end{aligned}
$$

If we set $Q=0$, Eqs. (6) - (8) reduce to the harmonic metric of a moving Schwarzschild black hole with velocity $v$

$$
\begin{aligned}
g_{00}= & -\frac{\gamma^{2}(1+\Phi)}{1-\Phi}+\boldsymbol{v}^{2} \gamma^{2}(1-\Phi)^{2}+\frac{\gamma^{2} \Phi^{2}(1-\Phi)}{1+\Phi} \frac{(\boldsymbol{v} \cdot \boldsymbol{X})^{2}}{R^{2}} \\
g_{0 i}= & v_{i} \gamma^{2}\left[\frac{1+\Phi}{1-\Phi}-(1-\Phi)^{2}\right]-\frac{\gamma \Phi^{2}(1-\Phi)}{1+\Phi}\left[\frac{X_{i}(\boldsymbol{v} \cdot \boldsymbol{X})}{R^{2}}+\frac{v_{i}(\gamma-1)(\boldsymbol{v} \cdot \boldsymbol{X})^{2}}{\boldsymbol{v}^{2} R^{2}}\right] \\
g_{i j}= & (1-\Phi)^{2} \delta_{i j}+\frac{\Phi^{2}(1-\Phi)}{R^{2}(1+\Phi)}\left[X_{i}+\frac{v_{i}(\gamma-1)(\boldsymbol{v} \cdot \boldsymbol{X})}{\boldsymbol{v}^{2}}\right]\left[X_{j}+\frac{v_{j}(\gamma-1)(\boldsymbol{v} \cdot \boldsymbol{X})}{\boldsymbol{v}^{2}}\right] \\
& +v_{i} v_{j} \gamma^{2}\left[(1-\Phi)^{2}-\frac{1+\Phi}{1-\Phi}\right]
\end{aligned}
$$

which are the extension of the exact metric [14] for a Schwarzschild black hole with $\boldsymbol{v}=v \boldsymbol{e}_{1}$. Here $R$ is also equal to $\sqrt{X_{1}^{2}+X_{2}^{2}+X_{3}^{2}}$. It is worth pointing out that Eqs. (9) - (11), to the first post-Minkowskian approximation, are in agreement with the gravitational Liénard-Wiechert retarded solution [3].

\section{DYNAMICS OF PARTICLE IN THE WEAK-FIELD LIMIT}

As an application, we apply the harmonic metric to derive the post-Newtonian dynamics of a neutral and non-relativistic particle in the far field of the moving Reissner-Nordström black hole. 
First, we expand Eqs. (6) - (8) up to an order of $1 / R^{2}$

$$
\begin{aligned}
& g_{00}=-1-2\left(1+2 v^{2}\right) \Phi-2 \Phi^{2}-\frac{Q^{2}}{R^{2}}, \\
& g_{0 i}=4 v_{i} \Phi, \\
& g_{i j}=(1-2 \Phi) \delta_{i j},
\end{aligned}
$$

where the velocity of the black hole has also been assumed to be non-relativistic, i.e., $\gamma \simeq 1$. After tedious but straightforward calculations, up to the order of $\bar{v}^{4} / \bar{r}$ ( $\bar{v}$ and $\bar{r}$ denote typical values

of velocity and separation of a system of particles, respectively), we can obtain the equation of motion of a massive particle as follow

$$
\frac{d \boldsymbol{u}}{d t}=-\nabla\left(\Phi+2 v^{2} \Phi+2 \Phi^{2}+\frac{Q^{2}}{2 R^{2}}\right)-\frac{\partial \boldsymbol{\zeta}}{\partial t}+\boldsymbol{u} \times(\nabla \times \boldsymbol{\zeta})+3 \boldsymbol{u} \frac{\partial \Phi}{\partial t}+4 \boldsymbol{u}(\boldsymbol{u} \cdot \nabla) \Phi-\boldsymbol{u}^{2} \nabla \Phi
$$

where $\boldsymbol{u}$ denotes the velocity of the particle, and $\zeta=4 v \Phi$. When the charge of the black hole vanishes, this equation reduces to the post-Newtonian dynamics of a non-relativistic particle in the field of a moving Schwarzschild black hole [14, 18].

\section{CONCLUSION}

The metric in harmonic coordinates plays an important role in the post-Newtonian dynamics and gravitational wave radiation. In this work we obtain the exact metric for a moving ReissnerNordström black hole via applying a Lorentz boost to the Reissner-Nordström metric in the harmonic coordinates. This method can avoid directly solving the Einstein field equations for a moving gravitational source. Based on this metric, we derive the post-Newtonian dynamics of a non-relativistic particle. This metric can also be used to calculate the deflection and time delay of light passing by a non-static Reissner-Nordström black hole, as well as Hawking radiation of the black hole.

\section{ACKNOWLEDGMENTS}

This work was supported in part by the Program for New Century Excellent Talents in University (Grant No. NCET-10-0702), the National Basic Research Program of China (973 Program) (Grant No. 2013CB328904), and the Ph.D. Foundation of the Ministry of Education of China 
(Grant No. 20110184110016).

[1] K. H. Look, C. L. Tsou and H. Y. Kuo, Acta Phys. Sin. 23, 225 (1974).

[2] T. Pyne and M. Birkinshaw, Astrophys. J. 415, 459 (1993).

[3] S. M. Kopeikin and G. Schäfer, Phys. Rev. D 60, 124002 (1999).

[4] M. Sereno, Phys. Lett. A 305, 7 (2002).

[5] S. M. Kopeikin and B. Mashhoon, Phys. Rev. D 65, 064025 (2002).

[6] M. Q. Miao, S. J. Qing and L. C. An, Acta Phys. Sin. 7, 049 (2003).

[7] M. Sereno, Mon. Not. R. Astron. Soc. 359, L19 (2005).

[8] M. Sereno, Mon. Not. R. Astron. Soc. 380, 1023 (2007).

[9] S. M. Kopeikin and V. V. Makarov, Phys. Rev. D 75, 062002 (2007).

[10] C. Bonvin, Phys. Rev. D 78, 123530 (2008).

[11] S. C. Novati, M. Dall'Ora et al., Astrophys. J. 717, 987 (2010).

[12] S. Zschocke and S. A. Klioner, Class. Quantum Grav. 28, 015009 (2011).

[13] O. Wucknitz and U. Sperhake, Phys. Rev. D 69, 063001 (2004).

[14] G. He and W. Lin, Commun. Theor. Phys. 61, 270 (2014).

[15] G. He and W. Lin, Int. J. Mod. Phys. D 23, 1450031 (2014).

[16] G. He, C. Jiang and W. Lin, under review (2014).

[17] W. Lin and C. Jiang, Phys. Rev. D 89, 087502 (2014).

[18] S. Weinberg, Gravitation and Cosmology: Principles and Applications of the General Theory of Relativity, Wiley, New York (1972). 